\title{
Pesquisa de fatores que influenciam na melhoria de processo de software em empresas do estado de Santa Catarina
}

\author{
Pablo Schoeffel \\ UDESC - Universidade do Estado de Santa \\ Catarina \\ pablo.schoeffel@udesc.br
}

\author{
Fabiane Barreto Vavassori Benitti \\ UNIVALI - Universidade do Vale do Itajaí \\ fabiane.benitti@univali.br
}

\begin{abstract}
Resumo
Este artigo descreve a execução e resultados descritivos de uma pesquisa realizada em forma de survey com 81 empresas de desenvolvimento de software do estado de Santa Catarina, a qual identifica a existência ou não de diversos fatores relacionados à melhoria de processo de software, divididos nos grupos de fatores: organizacionais, processo, produto, projeto, mercado/cliente, pessoal e melhoria de processo.
\end{abstract}

\section{Abstract}

This article describes the implementation and results of a survey with 81 software development companies in the state of Santa Catarina, which identified the existence of several factors related to improving software process, divided into six factors groups: organizational, process, product, project, customer/market, people and process improvement.

\section{Introdução}

Uma preocupação recorrente das empresas é pela entrega de melhores produtos e serviços, em um prazo cada vez mais curto e com orçamentos reduzidos, buscando, portanto, o aumento da qualidade do processo e do produto [1]

Segundo Deming [2] , um dos princípios da qualidade é procurar a melhoria contínua do processo produtivo, melhorando a qualidade e reduzindo custos.

Santa Catarina é um pólo de empresas de desenvolvimento de software, sendo que grande parte das empresas, seguindo a tendência nacional, são micro e pequenas, existindo um número pequeno de MPEs que possuem alguma certificação ou avaliação formal em melhoria de processo [3] .

Ao mesmo tempo, os métodos/modelos existentes talvez não se adaptem à realidade de grande parte das empresas, principalmente de micro e pequenas [4] [5] e esse pode ser um motivo pela não adesão a programas de melhoria de processos.

Portanto, existem diversos fatores organizacionais, pessoais, de processo e outros que supostamente podem influenciar no sucesso ou no fracasso da adoção de um modelo de melhoria de processo [7] .

Nesse contexto, é necessário identificar quais são os motivos ou os fatores que podem influenciar no processo de melhoria, facilitando ou impedindo a adoção dos modelos existentes e servindo como base para adaptação de novos modelos.

Visando identificar esses fatores, as práticas e modelos utilizados e a percepção quanto à SPI (Software Process Improvement) das empresas de Santa Catarina, esse artigo descreve os resultados de uma pesquisa sistemática, em forma de survey, realizada com 81 empresas do estado. O objetivo desse artigo é mostrar, de forma descritiva, os resultados para cada um dos fatores pesquisados.

As próximas seções deste artigo estão organizadas da seguinte maneira: na seção 2 são abordados detalhes da pesquisa e a metodologia adotada na realização da mesma. Na seção 3 os resultados são apresentados. Por fim, na seção 4 as conclusões.

\section{A Pesquisa}

Esta seção descreve os detalhes da pesquisa, que foi realizada com empresas de desenvolvimento de software do estado de Santa Catarina no período de Março a Julho de 2010.

\subsection{Escopo}

O principal foco deste estudo está em micro e pequenas organizações, apesar de serem pesquisadas também médias e grandes empresas (MGEs) para efeito de comparações. Não são consideradas pequenas unidades dentro de uma empresa maior, pois estas têm algumas características diferenciais, como, por exemplo, tipicamente acesso a escritório de gerenciamento de projetos (PMO), equipe de processo de engenharia de software, mais recursos financeiros, etc.

Em adição, o foco do estudo são as empresas cujo principal negócio é o desenvolvimento de software. Portanto, não são consideradas, por exemplo, empresas que tenham como principal negócio a consultoria de software, o fornecimento de suporte ou atendimento ao usuário.

Considerando-se a abrangência geográfica, a pesquisa é realizada com micro, pequenas, médias e grandes empresas do estado de Santa Catarina/Brasil.

A pesquisa foi enviada para 494 empresas, as quais foram mapeadas por esse trabalho, através da compilação das diversas fontes encontradas (associações 
de empresas, incubadoras, entidades da área, sindicatos, universidades, lista telefônica e pesquisas na Internet), sendo que retornaram 81 questionários válidos preenchidos.

A Figura 2 mostra o percentual das respostas por região.

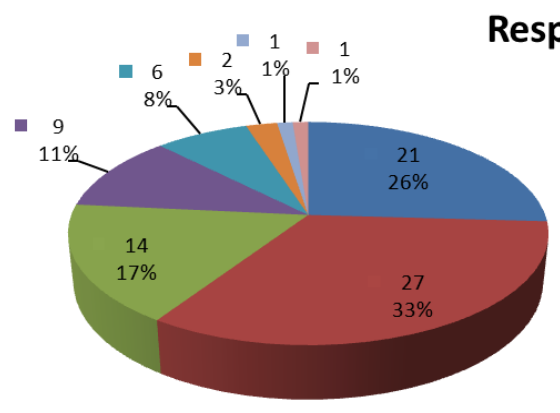

- Litoral

घ Vale do Itajaí

n Nordeste

- Oeste

nul

- Planalto Serrano

Meio Oeste

- Planalto Norte

Figura 2: Respostas por região de SC

\subsection{Fatores e Questionário}

Através de pesquisa bibliográfica foram encontrados diversos trabalhos e pesquisas descrevendo casos de melhoria de processo em pequenas empresas, assim como de adaptação ou criação de novos frameworks, métodos ou modelos específicos para MPEs.

Muitas pesquisas encontradas relatam casos de implantação de modelos de melhoria de processo ou então fatores de sucesso ou fracasso nas implantações. A Tabela 1 mostra 14 dessas pesquisas que contribuíram na execução dessa pesquisa.

\begin{tabular}{|l|l|}
\hline Fonte & \multicolumn{1}{|c|}{ Resumo } \\
\hline Paulk (1998)[6] & $\begin{array}{l}\text { Utilização de CMM para pequenas } \\
\text { organizações. }\end{array}$ \\
\hline $\begin{array}{l}\text { Stelzer e Mellis } \\
\text { (1999) [8] }\end{array}$ & $\begin{array}{l}\text { Pesquisa em 56 empresas de fatores } \\
\text { que afetam numa iniciativa de SPI. }\end{array}$ \\
\hline Butt (2000) [9] & $\begin{array}{l}\text { Dissertação que identifica fatores } \\
\text { que afetam SPI. }\end{array}$ \\
\hline $\begin{array}{l}\text { Beecham, Hall } \\
\text { e Reiner (2003) } \\
\text { [7] }\end{array}$ & $\begin{array}{l}\text { Pesquisa com profissionais de 12 } \\
\text { empresas que identifica problemas } \\
\text { na adoção de SPI. }\end{array}$ \\
\hline $\begin{array}{l}\text { Dyba (2002) } \\
\text { [10] }\end{array}$ & $\begin{array}{l}\text { Pesquisa com 120 empresas que } \\
\text { identifica fatores de sucesso em SPI. }\end{array}$ \\
\hline $\begin{array}{l}\text { Nasir, Ahmad e } \\
\text { Hassan (2008) } \\
\text { [11] }\end{array}$ & $\begin{array}{l}\text { Survey com profissionais de 29 } \\
\text { empresas para identificar fatores de } \\
\text { resistência à mudança em SPI. }\end{array}$ \\
\hline $\begin{array}{l}\text { Johnson e } \\
\text { Brodman } \\
\text { (1997) [5] }\end{array}$ & $\begin{array}{l}\text { Relato de experiência baseado no } \\
\text { trabalho em 200 empresas, } \\
\text { identificando problemas em } \\
\text { pequenas organizações adotar o } \\
\text { CMM. }\end{array}$ \\
\hline $\begin{array}{l}\text { O’Connor e } \\
\text { Colemann } \\
\text { (2007) [12] }\end{array}$ & $\begin{array}{l}\text { Estudo da adoção de SPI na prática } \\
\text { com 21 empresas. }\end{array}$ \\
\hline Jalote (2001) & Relato de experiência sobre fatores \\
\hline
\end{tabular}

\begin{tabular}{|l|l|}
\hline$[13]$ & $\begin{array}{l}\text { que facilitaram empresas em adotar } \\
\text { CMMI níveis 4 e 5. }\end{array}$ \\
\hline $\begin{array}{l}\text { Laporte, } \\
\text { Alexandre e } \\
\text { Renault (2008) } \\
{[3]}\end{array}$ & $\begin{array}{l}\text { Estudo realizado em 32 países para } \\
\text { identificar problemas na adoção de } \\
\text { padrões. }\end{array}$ \\
\hline $\begin{array}{l}\text { Desharnais, } \\
\text { Zarour e April } \\
\text { (2008) [14] }\end{array}$ & $\begin{array}{l}\text { Identificação de pontos fracos em } \\
\text { micro empresas com relação à SPI. }\end{array}$ \\
\hline $\begin{array}{l}\text { Brietzke e } \\
\text { Rabelo (2006) } \\
{[15]}\end{array}$ & $\begin{array}{l}\text { Pesquisa com 36 profissionais para } \\
\text { identificar fatores de resistência a } \\
\text { SPI, no estado do Rio Grande do Sul. }\end{array}$ \\
\hline $\begin{array}{l}\text { Cater-Steel } \\
\text { (2004) [16] }\end{array}$ & $\begin{array}{l}\text { Tese que descreve a realização de } \\
\text { um survey com 209 respostas para } \\
\text { identificar fatores de influência em } \\
\text { SPI numa região da Austrália. }\end{array}$ \\
\hline $\begin{array}{l}\text { Goldenson e } \\
\text { Herbsleb } \\
\text { (1995) [17] }\end{array}$ & $\begin{array}{l}\text { Pesquisa que identifica fatores de } \\
\text { barreira e sucesso em empresas que } \\
\text { adotaram CMMI. }\end{array}$ \\
\hline
\end{tabular}

Tabela 1: Resumo do estado da arte

Portanto, verifica-se que somente uma pesquisa similar foi encontrada no Brasil e ainda, das demais pesquisas, poucas buscam identificar efetivamente os fatores que podem influenciar a melhoria de processo nas empresas de software.

A partir da análise e síntese desses 14 desses trabalhos (Tabela 1), chegou-se a uma lista de fatores que podem influenciar a melhoria de processo de software.

Esses fatores geralmente são classificados como: fatores de sucesso, fatores de impacto ou fatores de influência na adoção de SPI, sendo que não foi encontrada uma classificação padronizada.

Essa sessão apresenta uma síntese desses fatores, baseada nos trabalhos pesquisados, visando abranger de maneira ampla os aspectos que podem influenciar na melhoria de processo.

Esses fatores foram classificados em sete grupos: organizacionais, produtos ou serviços, clientes/mercado, processo, projeto, pessoal e melhoria de processo (Tabela 2).

\begin{tabular}{|l|l|}
\hline \multicolumn{2}{|c|}{ Organizacionais } \\
\hline $\begin{array}{l}\text { Estrutura } \\
\text { Organizacional }\end{array}$ & Número de empregados \\
\hline Número de papéis & Infra-estrutura \\
\hline Resistência à mudança & Cultura organizacional \\
\hline $\begin{array}{l}\text { Definição de } \\
\text { responsabilidades/ } \\
\text { papéis }\end{array}$ & Comunicação dos \\
\hline $\begin{array}{l}\text { Orçamento/Recursos } \\
\text { financeiros }\end{array}$ & $\begin{array}{l}\text { Alinhamento de } \\
\text { objetivos de negócio }\end{array}$ \\
\hline Indivíduos-chave & Subcontratação \\
\hline Estágio de crescimento & $\begin{array}{l}\text { Necessidade } \\
\text { criatividade e inovação }\end{array}$ \\
\hline \multicolumn{2}{|c|}{ Produtos/serviços } \\
\hline Tipos de produtos/ & Nível de qualidade dos \\
\hline
\end{tabular}




\begin{tabular}{|c|c|}
\hline aplicações & produtos \\
\hline \multicolumn{2}{|c|}{ Clientes/mercado } \\
\hline $\begin{array}{lr}\text { Exigência } & \text { de } \\
\text { certificação/ } & \text { orientação } \\
\text { à exportação } & \\
\end{array}$ & $\begin{array}{l}\text { Setor/domínio } \\
\text { indústria }\end{array}$ \\
\hline \multicolumn{2}{|c|}{ Processo } \\
\hline \multicolumn{2}{|c|}{$\begin{array}{l}\text { Formalismo do processo (certificação e uso de } \\
\text { documentação e processos) }\end{array}$} \\
\hline \multicolumn{2}{|c|}{ Projeto } \\
\hline $\begin{array}{l}\text { Tamanho da equipe de } \\
\text { projeto }\end{array}$ & $\begin{array}{l}\text { Quantidade de projetos } \\
\text { simultâneos }\end{array}$ \\
\hline \multicolumn{2}{|c|}{ Tamanho/Complexidade/Duração do projeto } \\
\hline \multicolumn{2}{|c|}{ Pessoal } \\
\hline $\begin{array}{l}\text { Competência/ } \\
\text { Experiência }\end{array}$ & Rotatividade da equipe \\
\hline $\begin{array}{l}\text { Comprometimento } \mathrm{e} \\
\text { motivação da equipe }\end{array}$ & $\begin{array}{l}\text { Comprometimento da } \\
\text { gerência }\end{array}$ \\
\hline \multicolumn{2}{|c|}{ Melhoria de processo } \\
\hline $\begin{array}{l}\text { Competência e acesso a } \\
\text { especialistas em SPI }\end{array}$ & Retorno do Investimento \\
\hline $\begin{array}{l}\text { Disponibilidade de } \\
\text { tempo }\end{array}$ & $\begin{array}{l}\text { Capacidade de } \\
\text { gerenciamento }\end{array}$ \\
\hline $\begin{array}{l}\text { Recursos humanos e } \\
\text { financeiros }\end{array}$ & Objetivos da melhoria \\
\hline $\begin{array}{l}\text { Sistema de qualidade já } \\
\text { existente }\end{array}$ & $\begin{array}{l}\text { Adequação do modelo às } \\
\text { necessidades da } \\
\text { organização }\end{array}$ \\
\hline
\end{tabular}

Tabela 2: Fatores divididos em grupos

Para cada fator identificado, procurou-se criar uma ou mais perguntas no questionário utilizado pela pesquisa, de uma forma que cada fator pudesse ser verificado e analisado.

O questionário criado contém 55 perguntas, dividido nos mesmos 7 grupos utilizados para classificar os fatores e foi distribuído para a população identificada, tendo 81 respostas.

\subsection{Metodologia}

A pesquisa realizada nesse trabalho é classificada como survey on-line e descritivo/exploratório. Para realização deste tipo de pesquisa há diversas metodologias propostas na literatura (por exemplo, [18] e [19] ), porém a adotada por esse trabalho é a sugerida por Kasunic [20], por ser mais completa e concebida para a realidade de pesquisas em Engenharia de Software.

A Figura 3 resume a execução de cada uma das fases do survey. É importante observar que foi introduzida uma nova fase na metodologia originalmente proposta por Kasunic [20] para planejamento e gerenciamento dos riscos da pesquisa.

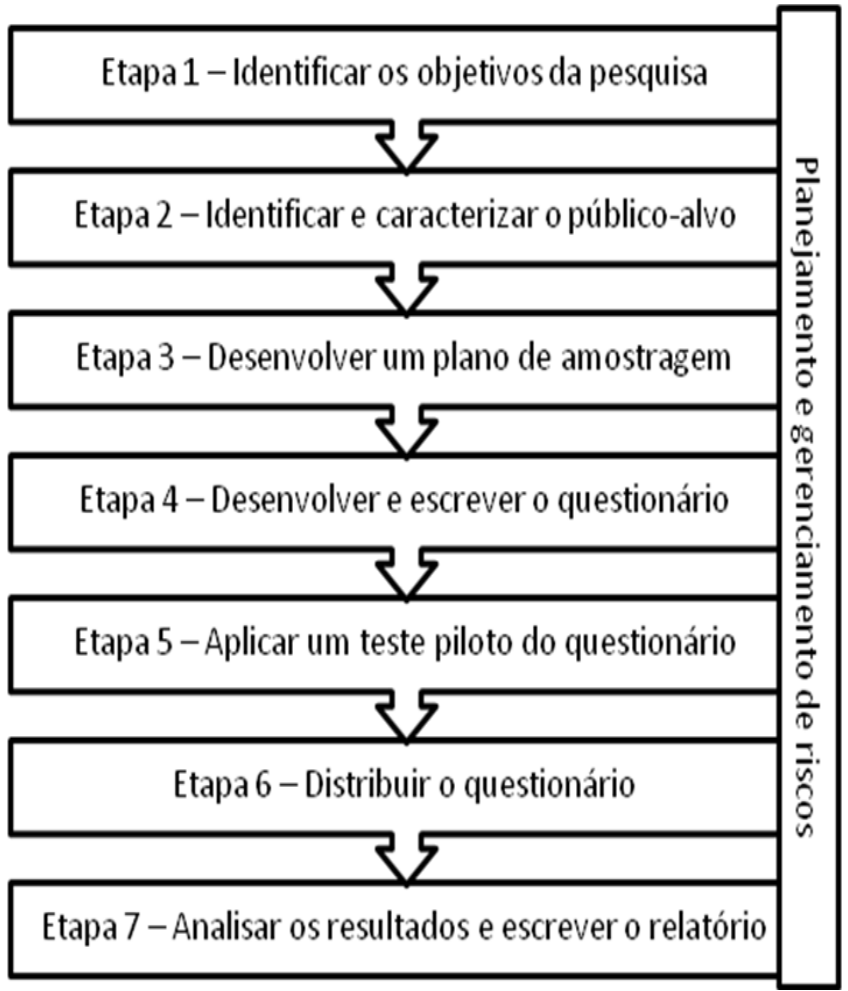

Figura 3. Fases do survey

A Tabela 3 mostra a relação dos principais riscos identificados e suas respectivas ações e situações.

\begin{tabular}{|l|l|l|}
\hline Risco & Ações & Situação \\
\hline $\begin{array}{l}\text { Não atingir o } \\
\text { número } \\
\text { mínimo de } \\
\text { empresas } \\
\text { definido na } \\
\text { amostragem }\end{array}$ & $\begin{array}{l}\text { 1) Reenviar o convite para } \\
\text { participação 1 mês após o } \\
\text { primeiro envio. }\end{array}$ & Executado \\
\cline { 2 - 3 } & $\begin{array}{l}\text { 2) Acompanhar } \\
\text { semanalmente o } \\
\text { andamento das respostas } \\
\text { e, caso identificado um } \\
\text { número aquém do } \\
\text { esperado, entrar em } \\
\text { contato telefônico com as } \\
\text { empresas. }\end{array}$ & Executado \\
\cline { 2 - 3 } & $\begin{array}{l}\text { 3) Uma semana após o } \\
\text { reenvio dos convites, caso } \\
\text { o número desejado não } \\
\text { seja obtido, agendar } \\
\text { visitas presenciais nas } \\
\text { empresas. }\end{array}$ & $\begin{array}{l}\text { Não } \\
\text { necessário }\end{array}$ \\
\cline { 2 - 3 } & $\begin{array}{l}\text { 4) Para servir como um } \\
\text { atrativo será estudado a } \\
\text { possibilidade de um } \\
\text { brinde para os } \\
\text { respondentes do } \\
\text { questionário (ex: pen } \\
\text { drive com o resultado da } \\
\text { pesquisa). }\end{array}$ & $\begin{array}{l}\text { Não } \\
\text { necessário }\end{array}$ \\
\hline
\end{tabular}




\begin{tabular}{|l|l|l|}
\hline & $\begin{array}{l}\text { 5) Se na data final não se } \\
\text { obter o número desejado, } \\
\text { reduzir o grau de } \\
\text { confiança e/ou aumentar o } \\
\text { erro amostral. }\end{array}$ & Executado \\
\hline $\begin{array}{l}\text { Não conseguir } \\
\text { estratificar as } \\
\text { respostas de } \\
\text { acordo com } \\
\text { região }\end{array}$ & $\begin{array}{l}\text { 1) Redefinir o escopo do } \\
\text { projeto para as regiões } \\
\text { com maior adesão. }\end{array}$ & $\begin{array}{l}\text { Não } \\
\text { necessário }\end{array}$ \\
\hline
\end{tabular}

Tabela 3: Lista de riscos da pesquisa

Os resultados são apresentados utilizando a estatística descritiva, com gráficos descritivos e descrição tabular.

\section{Resultados}

Essa seção descreve os principais resultados encontrados com a pesquisa, divididos em cada um dos grupos de fatores.

\subsection{Organizacionais}

Os fatores organizacionais identificam características administrativas, físicas e gerais do negócio da organização, sem ater-se ao processo de desenvolvimento de software em si.

Foi identificado que grande maioria das empresas (86\%) são empresas de desenvolvimento de software nacional, enquanto $6 \%$ são multinacionais e o restante outras classificações (mistas, departamentos, etc.). Seguindo a tendência nacional, foi identificado também que grande maioria das empresas (82\%) é micro e pequena ${ }^{1}$, conforme Figura 4 , sendo que a média de funcionários é de 41,54 por empresa.

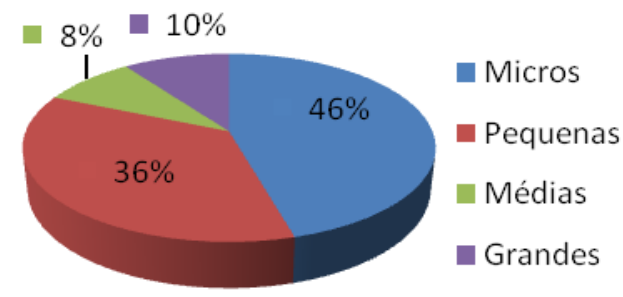

Já com relação ao faturamento, verificou-se que apenas $74 \%$ das empresas se enquadrariam no faturamento anual até R\$ 2,4 milhões, o que caracterizaria micro e pequena empresa, segundo o BNDS [21] e a legislação brasileira [22]

As empresas de SC, em sua maioria, desenvolvem software padrão com customizações para clientes (67\%), enquanto $22 \%$ desenvolvem sob encomenda e $5 \%$

${ }^{1}$ São consideradas micro e pequenas empresas aquelas que possuem até 49 funcionários [SEBRAE (2008)]. desenvolvem pacote sem customização (software de prateleira).

Com relação à terceirização, somente $10 \%$ utiliza mão-de-obra terceirizada. Em contrapartida, cerca de $59 \%$ das empresas fazem uso de componentes terceirizados.

Outro ponto pesquisado foi o estágio de crescimento das empresas, sendo que cerca de $26 \%$ estão em estágios iniciais (existência e sobrevivência), e as demais estão estabilizadas, em expansão ou em maturidade.

Verifica-se que cerca de $30 \%$ das empresas não possuem objetivos e estratégias de negócio definidas, sendo que somente $32 \%$ possuem os mesmos definidos formalmente e documentado.

Das $85 \%$ das empresas que possuem organograma definido, grande parte $(64 \%)$, principalmente micro e pequenas, possuem até 3 níveis hierárquicos, enquanto $17 \%$ possuem de 4 a 6 níveis e o restante possui mais que 6 níveis hierárquicos, em sua totalidade representados por médias e grandes empresas.

Grande parte das empresas $(95 \%)$ confirma uma característica do setor de desenvolvimento de software que é o acúmulo de papéis. Do total, $51 \%$ das empresas confirmam que a maioria dos funcionários desempenha vários papéis, embora somente $47 \%$ das empresas possuam os papéis e cargos definidos e documentados.

Analisando os aspectos referentes à resistência da empresa a novos projetos e ideias, verifica-se que em cerca de $47 \%$ das empresas sempre existe uma boa aceitação de novos projetos pelos colaboradores, porém $62 \%$ confirmam que muitos ou alguns desses projetos não são finalizados.

Com relação ao suporte pós venda, aproximadamente $53 \%$ das empresas realiza através de uma equipe específica, enquanto $48 \%$ realiza através da própria equipe de desenvolvimento.

Dos meios de comunicação, os mais utilizados diariamente são e-mail $(77 \%)$, ferramenta de gestão de atividades/tarefas (55\%), mensagens instantâneas (52\%), telefone $(45 \%)$ e conversa pessoal (43\%).

Quanto à localização física, a grande maioria das empresas (72\%) está num mesmo ambiente ou andar, aumentando para $84 \%$ se considerar o mesmo prédio e andares diferentes.

\subsection{Produtos ou serviços}

Esses fatores referem-se às características dos produtos ou serviços prestados pela organização.

Detalhando o tipo de aplicação dos produtos, podemse destacar os tipos de aplicação constantes da Tabela 4.

\begin{tabular}{|l|l|l|}
\hline Tipo de Aplicação & Nro. & Perc. \\
\hline
\end{tabular}




\begin{tabular}{|l|c|c|}
\hline Gestão integrada/ERP & 40 & $49,38 \%$ \\
\hline Automação comercial & 25 & $30,86 \%$ \\
\hline $\begin{array}{l}\text { Gestão do relacionamento } \\
\text { com cliente/CRM }\end{array}$ & 20 & $24,69 \%$ \\
\hline Websites & 19 & $23,46 \%$ \\
\hline Automação industrial & 18 & $22,22 \%$ \\
\hline Comércio eletrônico & 15 & $18,52 \%$ \\
\hline Contabilidade & 13 & $16,05 \%$ \\
\hline Gestão de conteúdo & 12 & $14,81 \%$ \\
\hline Administração de RH & 10 & $12,35 \%$ \\
\hline Automação de escritórios & 10 & $12,35 \%$ \\
\hline
\end{tabular}

Tabela 4: Empresas por tipo de aplicação

Somente $16 \%$ das empresas possuem alguma estimativa do tamanho de seus produtos. Ao mesmo tempo, grande maioria considera que seus produtos possuem complexidade média ou alta, com alguns ou muitos algoritmos complexos que demandem conhecimento técnico avançado. Ainda, 51\% das empresas consideram que seus produtos necessitam de inovações, porém não é crítico para o sucesso do mesmo, sendo que $31 \%$ consideram as inovações críticas e necessárias constantemente.

Com relação a aspectos de qualidade necessários para seus produtos, os aspectos considerados mais cruciais são (Tabela 5):

\begin{tabular}{|l|c|c|}
\hline Aspecto & Empresas & Perc. \\
\hline Confiabilidade & 61 & $75,31 \%$ \\
\hline Funcionalidade & 60 & $74,07 \%$ \\
\hline Usabilidade & 47 & $58,02 \%$ \\
\hline Eficiência & 34 & $41,98 \%$ \\
\hline
\end{tabular}

Tabela 5: Aspectos cruciais de qualidade

Em contrapartida, alguns aspectos são considerados não relevantes por muitas empresas (Tabela 6):

\begin{tabular}{|l|c|c|}
\hline Aspecto & Empresas & Perc. \\
\hline Portabilidade & 38 & $46,91 \%$ \\
\hline Testabilidade & 11 & $13,58 \%$ \\
\hline Reusabilidade & 11 & $13,58 \%$ \\
\hline
\end{tabular}

Tabela 6: Aspectos não relevantes de qualidade

\subsection{Clientes/mercado}

Refere-se às características do cliente e forma de comercialização do produto.

Quanto à área de atuação do produto, podem-se destacar as seguintes (Tabela 7):

\begin{tabular}{|l|c|c|}
\hline Área & Empresas & Perc. \\
\hline Comércio & 35 & $43,21 \%$ \\
\hline Indústria & 33 & $40,74 \%$ \\
\hline Serviços & 31 & $38,27 \%$ \\
\hline $\begin{array}{l}\text { Mercado empresarial } \\
\text { privado }\end{array}$ & 26 & $32,10 \%$ \\
\hline
\end{tabular}

\begin{tabular}{|l|c|c|}
\hline Financeiro & 17 & $20,99 \%$ \\
\hline $\begin{array}{l}\text { Agropecuária/ } \\
\text { Agrobusiness }\end{array}$ & 15 & $18,52 \%$ \\
\hline Educação & 13 & $16,05 \%$ \\
\hline Saúde & 13 & $16,05 \%$ \\
\hline Transportes & 13 & $16,05 \%$ \\
\hline
\end{tabular}

Tabela 7: Áreas de atuação dos produtos

Com relação à comercialização, aproximadamente $16 \%$ das empresas exportam software e $44 \%$ participam de licitações, sendo que $22 \%$ participam frequentemente.

\subsection{Processo}

Os fatores de processo referem-se ao grau de definição, documentação e execução dos processos das áreas de melhoria.

Cerca de $53 \%$ das empresas possuem uma pessoa ou equipe responsável pela engenharia de processos de software. Nessas empresas, em média existem 2,19 pessoas para essa atividade.

Quanto às práticas adotadas pelas empresas, destacase a orientação a objetos (77\%), o uso de frameworks de desenvolvimento $(52 \%)$ e percebe-se uma divisão entre arquitetura cliente/servidor (48\%) e web-bas ed (41\%). Outro fator importante é a utilização de métodos ágeis, com 27\% das empresas utilizando SCRUM e 16\% utilizando Extreme Programming - XP, sendo que nas MPEs esse percentual é maior $(29 \%$ e $19 \%$, respectivamente).

Quanto à documentação adotada no processo de desenvolvimento, destacam-se os seguintes documentos (Tabela 8):

\begin{tabular}{|l|c|}
\hline Documento & Perc. \\
\hline $\begin{array}{l}\text { Especificação de requisitos (casos } \\
\text { de uso) }\end{array}$ & $75,31 \%$ \\
\hline Proposta de orçamento & $75,31 \%$ \\
\hline Cronograma do projeto & $66,67 \%$ \\
\hline Contratos e acordos & $64,20 \%$ \\
\hline Lista de requisitos & $55,56 \%$ \\
\hline Modelagem do software & $50,62 \%$ \\
\hline Orçamento do Projeto & $48,15 \%$ \\
\hline $\begin{array}{l}\text { Descrição do projeto para } \\
\text { comercialização }\end{array}$ & $46,91 \%$ \\
\hline Manuais & $46,68 \%$ \\
\hline Documentação de código & $44,44 \%$ \\
\hline
\end{tabular}

Tabela 8: Documentação de processo adotada

Em contrapartida, existem documentos que são raramente utilizados, conforme Tabela 9 abaixo.

\begin{tabular}{|l|l|}
\hline Documento & \% \\
\hline Plano de medição & $3,70 \%$ \\
\hline
\end{tabular}




\begin{tabular}{|l|l|}
\hline Plano de gerência de dados & $7,41 \%$ \\
\hline Plano de comunicação & $7,41 \%$ \\
\hline Documentação de baselines & $7,41 \%$ \\
\hline Plano de riscos & $9,88 \%$ \\
\hline $\begin{array}{l}\text { Estrutura analítica do projeto } \\
\text { (WBS) }\end{array}$ & $9,88 \%$ \\
\hline $\begin{array}{l}\text { Documento de acompanhamento } \\
\text { do projeto (Status Report) }\end{array}$ & $13,58 \%$ \\
\hline $\begin{array}{l}\text { Registro de lições aprendidas e } \\
\text { ações corretivas }\end{array}$ & $14,81 \%$ \\
\hline $\begin{array}{l}\text { Documento de abertura do projeto } \\
\text { (Project Charter) }\end{array}$ & $16,05 \%$ \\
\hline
\end{tabular}

Tabela 9: Documentação de processo não adotada

Com relação a indicadores de desempenho, percebese que poucos indicadores são utilizados pela maioria das empresas, conforme gráfico abaixo (Tabela 10).

\begin{tabular}{|l|c|}
\hline Documento & Perc. \\
\hline Data início e fim das atividades & $79,01 \%$ \\
\hline Número de defeitos/falhas & $35,80 \%$ \\
\hline Grau de satisfação do cliente & $56,79 \%$ \\
\hline Não-conformidades do processo & $22,22 \%$ \\
\hline $\begin{array}{l}\text { Indicadores de desempenho do } \\
\text { processo }\end{array}$ & $6,17 \%$ \\
\hline Quantidade de retrabalho & $37,04 \%$ \\
\hline Satisfação dos funcionários & $23,46 \%$ \\
\hline Esforço realizado x previsto & $55,56 \%$ \\
\hline Outro & $3,70 \%$ \\
\hline
\end{tabular}

Tabela 10: Indicadores de desempenho utilizados

Outro aspecto pesquisado foi quais as áreas de processo que as empresas efetivamente têm definidas e implementadas. Foram utilizadas as áreas de processo do MPS.BR [23] e abaixo (Tabela 11) são exibidos os percentuais de empresas que possuem o modelo do processo implantado ou executam o processo informalmente.

\begin{tabular}{|l|c|c|}
\hline Área de processo & Empresas & Perc. \\
\hline Gerência de projetos & 60 & $74,07 \%$ \\
\hline Gerência de requisitos & 57 & $70,37 \%$ \\
\hline Aquisição de & 29 & $35,80 \%$ \\
\hline $\begin{array}{l}\text { Gerência } \\
\text { configuração }\end{array}$ & 51 & $62,96 \%$ \\
\hline Garantia da qualidade & 31 & $38,27 \%$ \\
\hline Gerência de portfolio & 41 & $45,68 \%$ \\
\hline $\begin{array}{l}\text { Medição processo } \\
\text { Avaliação e melhoria } \\
\text { organizacional }\end{array}$ & 38 & $46,91 \%$ \\
\hline $\begin{array}{l}\text { Definição do processo } \\
\text { organizacional }\end{array}$ & & \\
\hline
\end{tabular}

\begin{tabular}{|l|c|c|}
\hline $\begin{array}{l}\text { Gerência de recursos } \\
\text { humanos }\end{array}$ & 41 & $50,62 \%$ \\
\hline $\begin{array}{l}\text { Gerência de } \\
\text { reutilização }\end{array}$ & 27 & $33,33 \%$ \\
\hline $\begin{array}{l}\text { Desenvolvimento dos } \\
\text { requisitos }\end{array}$ & 65 & $80,25 \%$ \\
\hline Integração do produto & 59 & $72,84 \%$ \\
\hline $\begin{array}{l}\text { Projeto e construção } \\
\text { do produto }\end{array}$ & 73 & $90,12 \%$ \\
\hline Validação & 67 & $82,72 \%$ \\
\hline Verificação & 43 & $53,09 \%$ \\
\hline $\begin{array}{l}\text { Desenvolvimento para } \\
\text { reutilização }\end{array}$ & 30 & $37,04 \%$ \\
\hline Gerência de riscos & 32 & $39,51 \%$ \\
\hline Gerência de Decisões & 67 \\
\hline
\end{tabular}

Tabela 11: Áreas de processo executadas

\subsection{Projeto}

Os fatores de projeto referem-se ao comportamento da empresa com relação às atividades do processo de gerência de projeto.

A média de duração dos projetos é de 209,29 dias, ou seja, aproximadamente 7 meses.

Em média, existem 2,91 equipes de projeto por empresa e cada equipe é composta de, em média, 4,04 pessoas.

Verificou-se que os únicos papéis presentes de forma quase unânime nas equipes de projeto são de analista de sistemas e programador, conforme Figura 5.

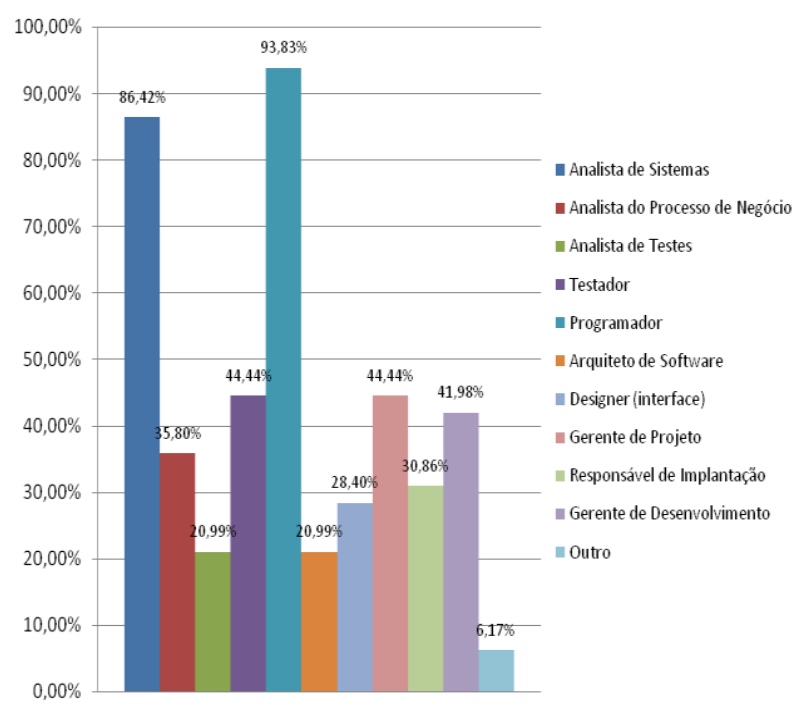

Figura 5. Papéis em projetos de software

Quanto aos processos de gerência de projetos, a maioria (cerca de 80\%) das empresas inicia, planeja e executa os projetos, porém um percentual menor monitora $(58 \%)$ e encerra $(63 \%)$ os mesmos. 
Quanto às áreas de conhecimento, as mais consideradas na prática são prazo, qualidade, custo e escopo, conforme Figura 6.

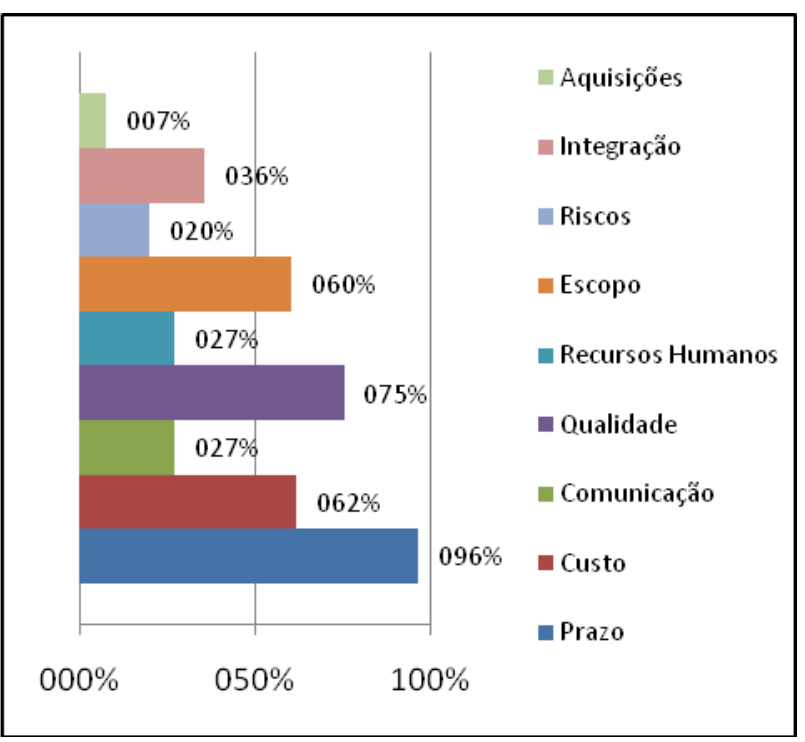

Figura 6. Áreas de conhecimento de GP

\subsection{Pessoal}

Os fatores pessoais estão relacionados às características e forma de gestão dos recursos humanos da organização.

Foi identificado que grande maioria das empresas depende do conhecimento de uma ou poucas pessoas, conforme Figura 7.

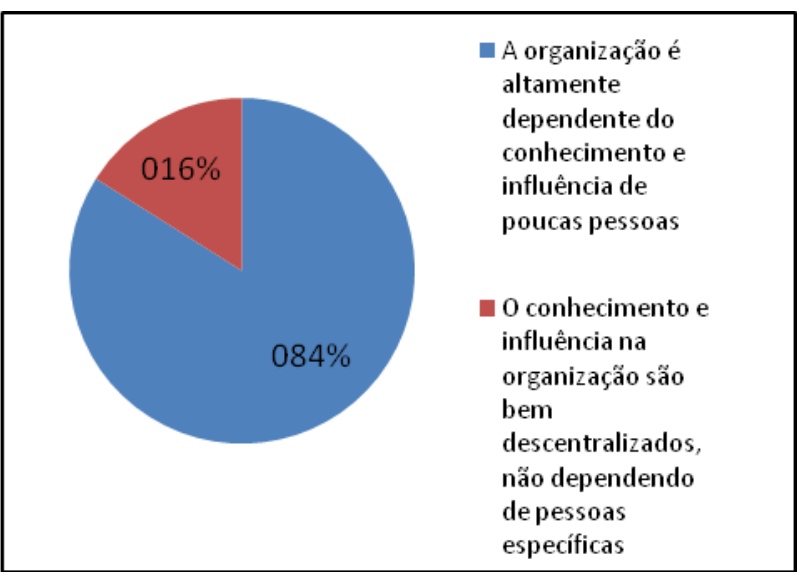

Figura 7. Dependência de pessoas

O nível de rotatividade de funcionários nas empresas também foi verificado e identificou-se que a maioria (57\%) teve uma rotatividade, nos últimos 2 anos, abaixo de $10 \%$ de seu quadro funcional, enquanto $21 \%$ das empresas tiveram rotatividade entre 10 e $25 \%$ e que $14 \%$ das empresas tiveram rotatividade superior a $25 \%$ de seu quadro funcional.
Outro fator identificado foi a participação frequente da alta gerência nos projetos da empresa, conforme Figura 8.

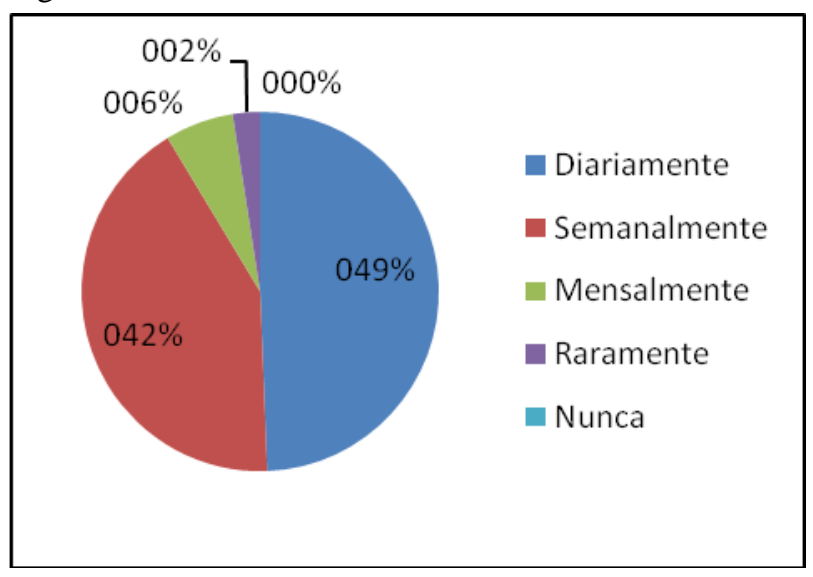

Figura 8. Envolvimento da alta gerência

Quanto à formação dos funcionários, existe um grande número de técnicos com formação superior na área (90\% das empresas possuem todos ou a maioria dos técnicos formados). Em contrapartida, existe um pequeno número de pessoas com certificações técnicas específicas $(30 \%$ das empresas não possuem certificação alguma e $54 \%$ possuem uma minoria dos funcionários com alguma certificação).

Quanto aos treinamentos realizados, as formas mais utilizadas são treinamentos internos e por iniciativa individual (fora do trabalho) e principalmente na área de tecnologia (treinamentos técnicos) conforme Figura 9.

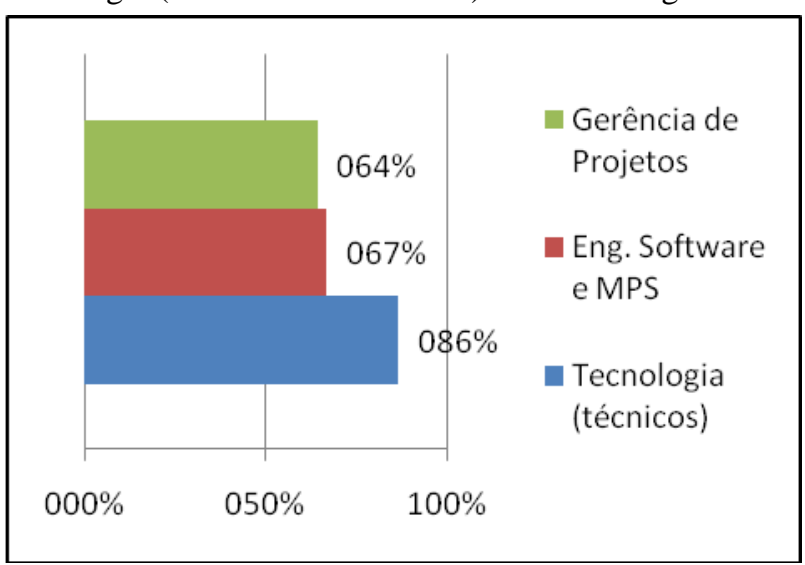

Figura 9. Tipos de treinamentos

\subsection{Melhoria de processo}

Esses fatores referem-se a como as empresas encaram os programas de melhoria, assim como os modelos/normas adotados pelas mesmas.

As empresas pesquisadas consideram que as metas de melhoria mais significativas são a expansão da faixa de mercado $(60,49 \%)$, aumento de produtividade $(48,15 \%)$ e aumento na satisfação dos clientes $(38,27 \%)$.

Foi constatado que apenas 7 empresas possuem certificação ou avaliação formal em melhoria de processo (Figura 10), sendo que uma delas possui 2 
certificações. Dessas, apenas uma é micro ou pequena (abaixo de 50 funcionários).

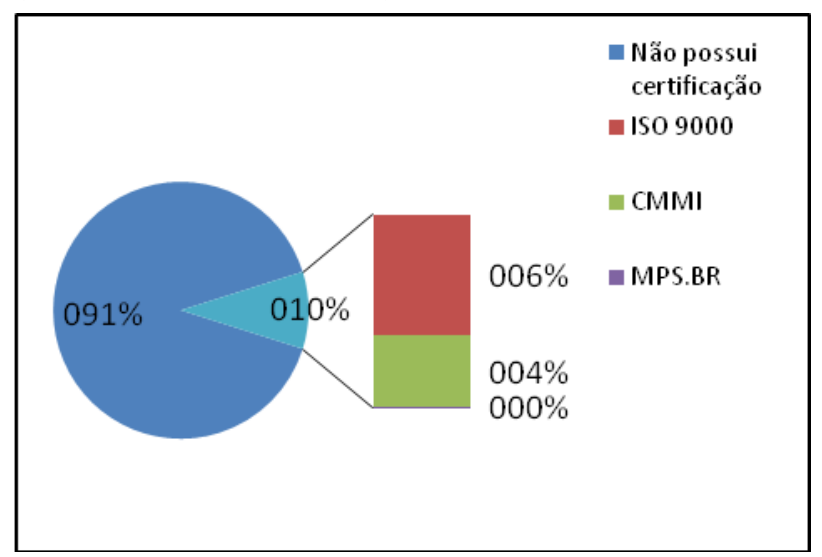

Figura 10. Empresas com certificação/avaliação

Apesar da grande maioria não ser certificada ou avaliada formalmente, existe um percentual significativo de empresas que está implantando ou pretender utilizar alguns modelos ou metodologias, conforme Tabela 12.

\begin{tabular}{|l|l|l|l|l|}
\hline \multicolumn{1}{|c|}{ Modelo/norma } & \multicolumn{2}{c|}{$\begin{array}{c}\text { Em } \\
\text { andamento }\end{array}$} & \multicolumn{2}{c|}{$\begin{array}{c}\text { Pretende } \\
\text { usar }\end{array}$} \\
\hline ISO 9000 & 2 & $2,47 \%$ & 16 & $19,75 \%$ \\
\hline ISO/IEC 12207 & 2 & $2,47 \%$ & 15 & $18,52 \%$ \\
\hline ISO/IEC 15504 & 2 & $2,67 \%$ & 16 & $19,75 \%$ \\
\hline CMMI & 2 & $2,67 \%$ & 20 & $24,69 \%$ \\
\hline MPS.BR & 4 & $4,94 \%$ & 26 & $32,10 \%$ \\
\hline ITIL & 3 & $3,70 \%$ & 13 & $16,05 \%$ \\
\hline COBIT & 2 & $2,47 \%$ & 8 & $9,88 \%$ \\
\hline OPM3/PMBOK & 8 & $9,88 \%$ & 16 & $19,75 \%$ \\
\hline
\end{tabular}

Tabela 12: Áreas de atuação dos produtos

Além disso, 5 empresas informaram já estar utilizando o guia PMBOK, 1 empresa o COBIT e 2 o ITIL.

Das empresas que não possuem certificação ou avaliação formal ainda, os motivos mais citados para isso estão relatados abaixo (Tabela 13).

\begin{tabular}{|l|l|}
\hline Motivo & \multicolumn{1}{|c|}{ Perc. } \\
\hline Não requerido pelo cliente & $41,10 \%$ \\
\hline Não requerido pela empresa & $23,29 \%$ \\
\hline Falta de suporte da alta gerência & $10,96 \%$ \\
\hline Falta de suporte do governo & $6,85 \%$ \\
\hline Falta de suporte da indústria & $0,00 \%$ \\
\hline Falta de recursos financeiros & $46,58 \%$ \\
\hline Falta de recursos pessoais & $45,21 \%$ \\
\hline Falta de ferramentas & $8,22 \%$ \\
\hline Implementação muito demorada & $16,44 \%$ \\
\hline Avaliação/Certificação muito demorada & $9,59 \%$ \\
\hline $\begin{array}{l}\text { Norma/modelo muito difícil e } \\
\text { burocrático }\end{array}$ & $32,88 \%$ \\
\hline Falta de orientação ou conhecimento & $32,88 \%$ \\
\hline Modelos não compatíveis com empresa & $26,03 \%$ \\
\hline
\end{tabular}

Tabela 13. Motivos para não certificação

Daquelas empresas que passaram pelo processo de melhoria e certificação ou avaliação, os principais problemas ou barreiras encontrados foram a resistência às mudanças das pessoas $(77,78 \%)$, falta de tempo e pessoas para as atividades (67\%), falta de material (33\%) e falta de envolvimento e comprometimento das pessoas (33\%). Além disso, essas mesmas empresas classificaram, numa forma geral, o processo de melhoria como bom ou muito bom, conforme Figura 11.

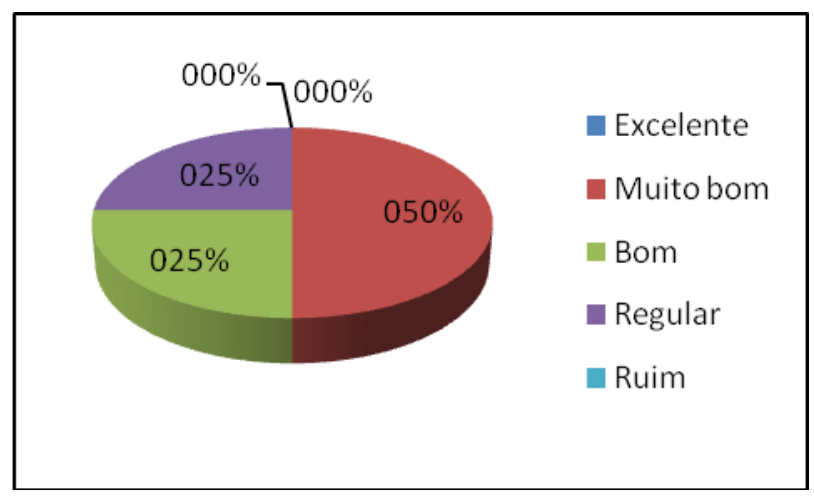

Figura 11. Satisfação do processo de melhoria

A grande maioria dessas empresas que implementaram SPI buscou consultoria externa (100\%) e instituições de ensino $(37,5 \%)$ para auxiliar no processo. $\mathrm{O}$ aspecto relatado que mais melhorou após o programa de melhoria foi o aumento da qualidade, sendo apontado por todas as empresas, e também o cumprimento de prazos e facilidade na execução das atividades, ambas apontado por metade das empresas, conforme Figura 12.

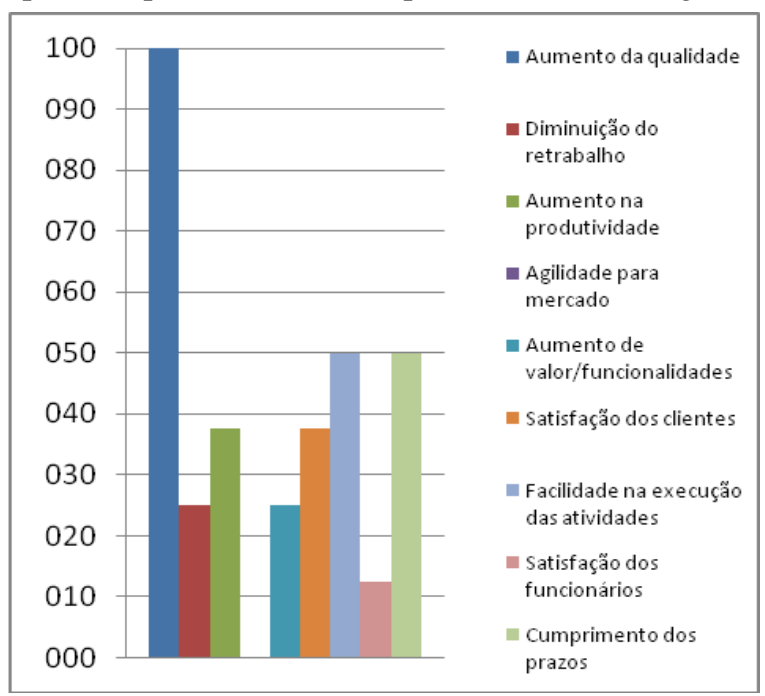

Figura 12. Aspectos de melhoria com o SPI

Outro ponto importante pesquisado foi a adequação das normas e modelos existentes à realidade das empresas, onde se verificou que a maioria das empresas acredita que os modelos precisam ser adaptados (Figura 13). 


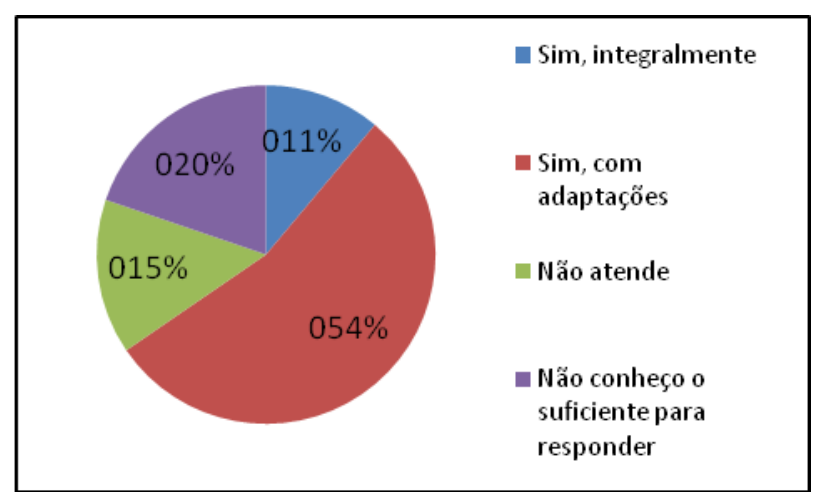

Figura 13. Adequação dos modelos

\section{Conclusões}

A pesquisa traça um perfil de um conjunto de empresas de desenvolvimento de software de Santa Catarina, identificando informações que podem ser relevantes para quaisquer pesquisas e ações de melhoria de processo nas mesmas.

O trabalho também demonstra que diversos aspectos assemelham-se à realidade de pesquisas nacionais ou mesmo internacionais, como a predominância de micro e pequenas empresas e o baixo índice de certificação/avaliação formal, principalmente de micro e pequenas empresas.

Além disso, a pesquisa identifica vários aspectos que podem influenciar no sucesso ou insucesso da implantação de um modelo de melhoria de processo de software e que podem ser levados em consideração para a implantação ou adaptação desse tipo de modelo.

Com relação à estrutura da empresa pode-se observar que grande maioria possui uma estrutura reduzida, com poucos níveis hierárquicos e poucos papéis na equipe, sendo que a maioria dos funcionários acaba acumulando papéis.

Com relação ao processo, verifica-se que é utilizada pouca documentação e são utilizadas poucas métricas pra monitoramento do processo.

Em contrapartida, a maioria das empresas identificam a necessidade de melhoria de qualidade, cumprimento de prazos e produtividade e aproximadamente uma em cada três empresas já implementou, está implementando ou pretende implementar algum modelo/norma de melhoria de processo.

Esses números enfatizam uma grande necessidade de aumentar a adesão de programas de melhoria, porém grande parte das empresas identifica que os modelos precisam ser adequados as suas realidades, além de mencionar problemas, como a falta de recursos financeiros e humanos.

A questão fundamental a respeito dos resultados do experimento é quão válidos são eles [24] Para isso, a pesquisa identificou cinco ameaças à validade: i) população identificada não representa população real; ii) tamanho da amostra não representa a população; iii) desvio nos resultados devido a fatores sem comprovação; iv) falta de entendimento de algumas perguntas; v) falta de conhecimento para entender e responder algumas perguntas.

Espera-se que esses resultados sirvam de apoio para novos estudos de criação e/ou adaptação de modelos de melhoria de processo em empresas de software e até mesmo como parâmetro para as próprias empresas e órgãos do setor realizarem ações nesse sentido.

\section{Referências}

[1] SEI - Software Engineering Institute, "Capability Maturity Model Integration for Development (CMMI-DEV)" Version 1.2. Ago. 2006. Disponível em: <http://www.sei.cmu.edu/cmmi/>. Acesso em: 22 set. 2008.

[2] E.W. Deming, "Qualidade: a revolução da administração“, Rio de Janeiro: Marques-Saraiva, 1990. 367 p.

[3] A. Renault, C. Laporte, and S. Alexandre, "Developing International Standards for Very Small Enterprise", IEEE Computer, vol. 41, nr 3, March 2008.

[4] C. G. Von Wangenheim, and I. Richardson, ",Why Are Small Software Organizations Differents?" IEEE Software, vol. 4, nr. 1, Jan 2007, pag. 18-22.

[5] D. L. Johnson, and J. G. Brodman, "Tailoring the CMMSM for Small Businesses, Small Organizations and Small Projects", IEEE Computer Society, n. 8. 1997

[6] M.C. Paulk, "Using the Software CMMI® in Small Organizations", Carnegie Mellon University, 1998.

[7] A. Rainer, S. Beecham, and T. Hall. "Software Process Improvement Problems in Twelve Software Companies: an empirical analysis", Empirical Software Engineering, v. 8, nro. $1, \quad$ mar/2003. Disponível em: <http://www.springerlink.com/content/hr397k6v15hw9311/ >. Acessado em: 21 set. 2008.

[8] D. Stelzer, and W. Mellis, "Success Factors of Organizational Change in Software Process Improvement", Software Process Improvement and Practice, vol. 4, 1999. Disponível <http://informationsmanagement.wirtschaft.tuilmenau.de/forschung/documents/successf.pdf >. Acessado em: 15 abr. 2009.

[9] A.J. Butt, BUTT, "Identifying Factors Affecting Software Process Improvement during Change", Tese de doutorado do curso de Engenharia de Software, Bleking Institute of Technology, 2007. Disponível em: <http://www.bth.se/fou/cuppsats.nsf/all/>. Acessado em: 21 set. 2008.

[10] T. Dyba, "Enabling Software Process Improvement: An Investigation of the Importance of Organizational Issues”, Empirical Software Engineering, vol. 7, nr. 4, Dez 2002, pag. 387-390.

[11] M.H.N. Nasir, N. H. Hassan, and R. Ahmad, "Resistance Factors in the Implementation of Software Process Improvement Project in Malaysia“, Journal of Computer Science, nro. 4, p. 211-219. 2008.

[12] G. Coleman, and R.V. O'Connor, “An Investigation of Barriers to the Adoption of Software Process Best Practice Models", $18^{\text {th }}$ Australasian Conference on Information Systems: Toowoomba. Dez. 2007.

[13] P. Jalote, "The Success of the SPI Efforts in India“, Software Quality Professional, vol. 3, No. 2, March 2001, pp. 36-40.

[14] A. April, J. Desharnais, and M. Zarour, "Very Small Enterprises (VSE) Quality Process Assessment". Disponível em: 
〈http://www.lrgl.uqam.ca/publications/pdf/1048.pdf〉. Acessado em: 22 abr. 2008.

[15] A. Rabelo, and J. Brietzke, "Resistance Factors in Software Processes Improvement", CLEI Eletronic Journal, vol 9, nro. 1. Junho, 2006.

[16] A.P. Caterr-steel, "An evaluation of Software Development Practice and Assessment-Based Process Improvement in Small Software Development Firms", Tese de doutorado do curso de Filosofia, Griffith University, 2004. Disponível em: <http://www4.gu.edu.au:8080/adtroot/public/adt-QGU20060110.180345/index.html>. Acessado em: 31 Ago. 2008.

[17] D.R. Goldenson, and J.D. Herbsleb, "After the Appraisal: a systematic survey of process improvement, its benefits, and factors that influence success", Technical Report - Software Engineering Institute. 1995.

[18] D. A. Dillman, "Mail and Internet Surveys: The Tailored Design Methods", Second edition. New York: Wiley, 2000.

[19] T. Punter et al. "Conducting On-line Surveys in Software Engineering", Proceedings of the 2003 International Symposium on Empirical Software Engineering (ISESE'03), 2003.

[20] M. Kasunic, "Designing an Effective Survey", Report of SEI - Carnegie Mellon University, 2005.

[21] Banco Nacional de Desenvolvimento Regional, "Porte de Empresa", Disponível em: <http://www.bndes.gov.br/clientes/porte/porte.asp>. Acessado em: 05 jul. 2008.

[22] Brasil. "Lei Complementar $\mathrm{N}^{\circ} 123$, de 14 de Dezembro de 2006. Institui o Estatuto Nacional da Microempresa e da Empresa de Pequeno Porte". Disponível em: <https://www.planalto.gov.br/ccivil_03/leis/lcp/lcp123.htm >. Acessado em: 01 jun. 2008.

[23] SOFTEX - Associação para Promoção da Excelência do Software Brasileiro. "MPS.BR - Melhoria de Processo do Software Brasileiro: guia geral", 2009. Disponível em: <www.softex.org.br>. Acessado em: 26 jun. 2009.

[24] D. Gurov, E. A. Amaral, and G. Travassos, "Introdução à Engenharia de Software Experimental", Relatório Técnico do Programa de Engenharia de Sistemas e Computação da COPPE/UFRJ, Rio de Janeiro, 2002. Disponível em: <http://sergio.dsc.upe.br/es/IntroducaoExperimentacao.pdf >. Acessado em: 20 ago. 2009. 